\title{
THE DIACETONE ACRYLAMIDE CROSSLINKING REACTION AND ITS INFLUENCE ON THE FILM FORMATION OF AN ACRYLIC LATEX
}

\author{
Dr. Nicola Kessel, Sun Chemical Ltd, Orpington, Kent, UK \\ Dr. Derek R. Illsley, Sun Chemical Ltd, Orpington, Kent, UK \\ Dr. Joseph L. Keddie, University of Surrey, Guildford, Surrey, UK
}

\begin{abstract}
Waterborne colloidal polymers (i.e. latex) represent a promising alternative to organic solvent-based systems in coatings applications. The development of mechanical strength and hardness is often enhanced by chemical crosslinking that creates a threedimensional network. If extensive crosslinking occurs within the particles prior to their coalescence, however, interdiffusion will be prevented. A weaker product will result. We have explored the inter-relationship between coalescence, crosslinking and surfactant exudation in an acrylic latex containing diacetone acrylamide, exploiting the "keto-hydrazide" crosslinking reaction. The complementary use of spectroscopic techniques on a model system determined that the crosslinking reaction yields an imine product, not an enamine as has been proposed in the literature. Gel fraction measurements were used to probe the rate of crosslinking and identified a slower rate in larger particles, suggesting that the transport of the crosslinking agent is ratelmiting. The keto-hydrazide reaction was found to be acid catalysed and favoured at lower water concentration. Measurement of the latex $\mathrm{pH}$ relative to the polymer mass fraction during film formation clarified the expected point of onset for crosslinking in relation to particle packing. Atomic force microscopy was used to follow surface levelling relative to the competing influence of crosslinking. The rate and total amount of surfactant exudation were found to be influenced by crosslinking, particle deformability (as determined by the temperature relative to the polymer glass transition temperature, $T_{\mathrm{g}}$ ), and the evaporation rate (as controlled by the relative humidity). There is evidence that surfactant exudation can be triggered by the particle deformation that occurs at film formation temperatures well above the $T_{\mathrm{g}}$.
\end{abstract}

\section{KEYWORDS}

Crosslinking, diacetone acrylamide, film formation, keto-hydrazide, surfactant.

\section{INTRODUCTION}

A growing interest in and uses for waterborne polymer coatings have been driven by increasing environmental pressures, especially the need to comply with legislation limiting volatile organic compounds and emissions, associated with the use of solvent borne polymer systems ${ }^{1,2,3,4}$. Waterborne colloidal polymers (i.e. "latex") are used in a wide range of applications, including adhesives, additives for paper, paints and coatings, printing inks, cosmetics, synthetic rubbers, floor polishes and waxes, sealants, and drug delivery systems ${ }^{4}$. Colloidal particles may be tailored to exhibit a desired morphology, composition, particle size distribution, surface groups, and molecular weight ${ }^{5}$. In turn, these particles can be manipulated during the film formation process in order to create coatings that meet the desired end-use 
requirements. Here, we consider the effects of several inter-related aspects of film formation ${ }^{4}$ on final film structure and properties.

The incorporation of crosslinking chemistry in waterborne coatings is recognised to provide a particularly effective means of enhancing the mechanical strength, chemical stability and solvent resistance of the final film ${ }^{6-11}$. Recently, a system based on the reaction of a carbonyl pendant group on the dispersed polymer backbone with a diamine, specifically where this amine is a dihydrazide, has been the subject of increased interest ${ }^{12,13,14}$. This chemistry, termed the keto-hydrazide reaction, offers the advantage of fast, ambient-temperature crosslinking in functionalized acrylic latex, when the dihydrazide is incorporated in the aqueous phase of the latex. Anecdotal evidence ${ }^{15}$ also suggests that an added benefit of keto-hydrazide chemistry, particularly in printing ink applications, is the enhancement of adhesion, possibly through hydrogen bonding at the substrate interface, or the formation of permanent covalent bonds between the dihydrazide and carbonyl groups at the treated polymer substrate surface.

Our system of interest consists of an acrylic latex containing diacetone acrylamide pendant groups (Figure 1A) on the polymer backbone that reacts with an adipic dihydrazide di-functional cross-linker (Figure 1B). It is conceivable that this reaction will yield either an imine or an enamine, or a mixture of both ${ }^{12,13,14,15,16}$. The precise mechanism has not been reported, and there has been speculation in the literature that there is an enamine product. [reference] This lack of clarity has motivated this present work.

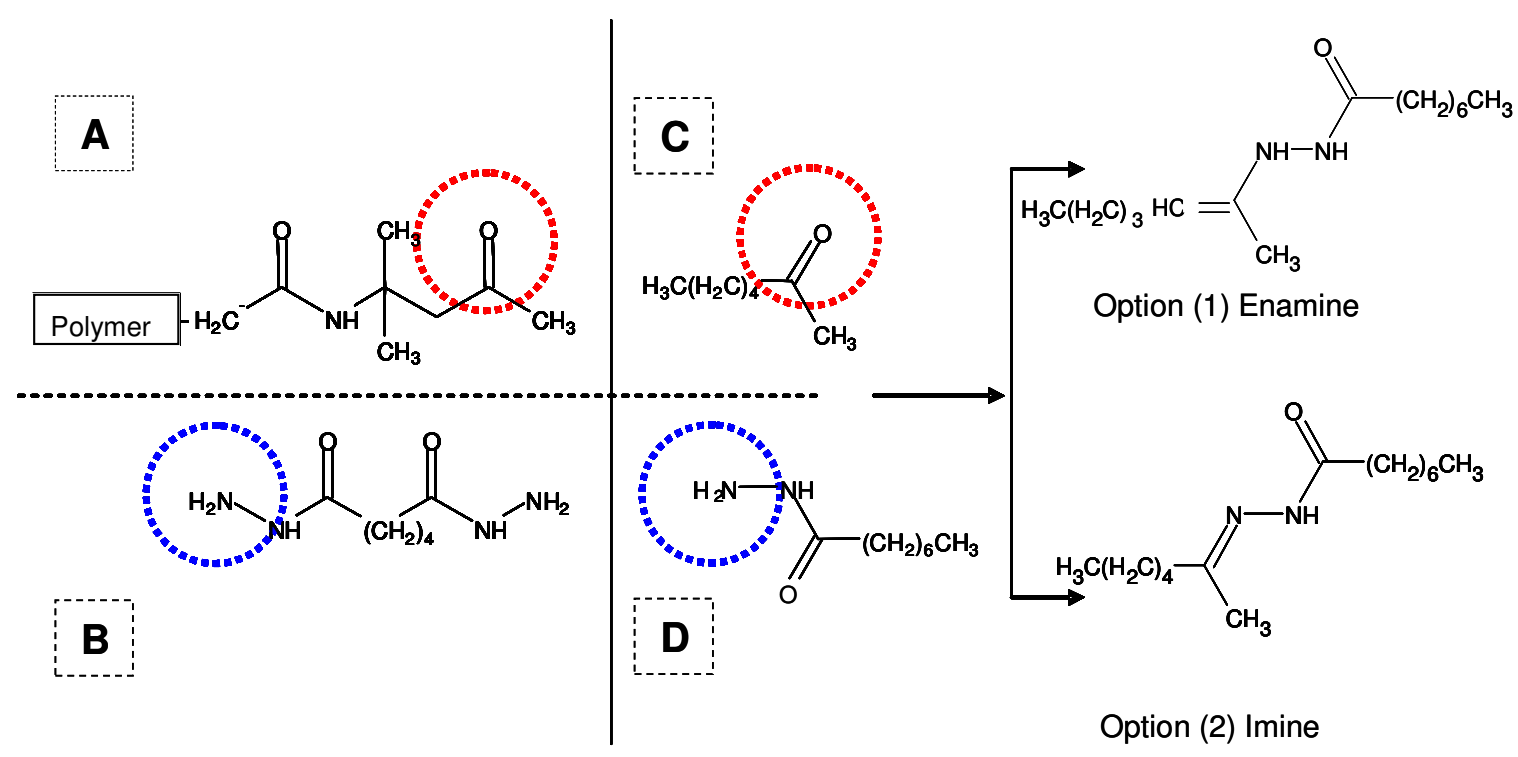

Figure 1: Molecular structure of the model reactants: (A):- Diacetone acrylamide pendant groups on the polymer backbone; (B):- Adipic dihydrazide cross-linker; (C):2-Heptanone, modelling the ketone group in the diacetone acrylamide pendant group; (D):- Octanoic hydrazide, modelling the amine group in adipic dihydrazide. Reaction between 2-Heptanone (C) and octanoic hydrazide (D) could yield option (1) an enamine, and/or option (2) an imine. 
An understanding of the fundamental reaction mechanism and kinetics is essential to optimise this coating formulation for specific applications and for the development of new materials. In this work we use model compounds (Figure $1 \mathrm{C}$ and 1D) to establish the product of the reaction, in order to simplify the spectroscopic analysis.

In waterborne systems the time in the film formation process at which crosslinking occurs can have a profound effect ${ }^{17-26}$. To achieve maximum film strength particles should remain relatively free of cross-links in the dispersion but undergo extensive crosslinking once they have formed a coating on the substrate. This is because molecular interdiffusion between neighbouring particles, which is essential for the generation of latex film strength, must take place prior to the crosslinking reaction ${ }^{23-}$ 29 . Strongly cross-linked particles are unable to inter-diffuse ${ }^{17,20,21}$. In systems having an external cross-linker that is dissolved in the aqueous phase, its partitioning character between the polymer and water imparts a further complication. If, as the film dries, this cross-linker does not readily dissolve within and uniformly distribute within the polymer particles, then localised crosslinking may result. Although the competing effects of crosslinking and interdiffusion in various waterborne systems have been studied, there are no such reports on keto-hydrazide coatings, until now. Moreover, we address the fact that $\mathrm{pH}$ evolves during film formation, and it has a catalytic influence on crosslinking, thus determining when the reaction develops as the film dries.

Note that the crosslinking reaction in our system can occur between DAAM groups within the same particle or it can occur at the interface between particles. Intra-particle crosslinking will increase the stiffness and strength, but without entanglements at particle/particle interfaces, the films will lack cohesion. Inter-particle crosslinking will generate more cohesion, however, even in the absence of entanglements and interdiffusion.

Through the years some strange and unusual observations of surfactant "islands" and "blobs" on coating surfaces have gone unexplained and remain mysterious ${ }^{30-35}$. It is known that the presence of surfactants profoundly influence the mechanical strength, durability, adhesion, blocking, gloss and permeability of the final film ${ }^{36-40}$. During this research it was discovered that the properties of the latex particles, the stages and conditions of film formation, and crosslinking all influence the rate of surfactant exudation. The processes are inter-related and should not be considered in isolation.

\section{EXPERIMENTAL}

\section{Reagents}

Butyl acrylate (BA), methyl methacrylate (MMA), diacetone acrylamide (DAAM), methacrylic acid (MA), styrene (St), acrylic acid (AA) ammonium persulphate (APS), adipic dihydrazide (ADH), octanoic hydrazide and 2-heptanone were used as supplied from Sigma Aldrich Chemicals. Sodium lauryl sulphate (Texapon K-12) was used as supplied from Henkel. Acetone, methanol (HPLC grade), water (HPLC grade), ammonium hydroxide, hydrochloric acid, and litmus, were used as supplied from Fisher Scientific Ltd. 


\section{Preparation of Latices}

The latices were prepared by a starve-feed emulsion polymerisation process using BA, MMA, DAAM, MAA and St monomers emulsified with sodium lauryl sulphate as the surfactant. APS was used as the initiator. The reaction flask (1 litre) was charged with deionised water and surfactant and heated to $80^{\circ} \mathrm{C}$ via a thermostatically-controlled heating mantle. Care was taken to avoid direct surface contact between the flask and heating mantle in order to prevent scorching of the flask contents.

A pre-prepared monomer "seed" mixture, containing all five monomers, was added to the flask, whilst stirring, and the temperature allowed to stabilise. Then an initiator "seed" was similarly added to the reaction vessel. The mixture was left for approximately 15 minutes to allow the latex "seed" to develop, before commencing the monomer feeds. Monomer was fed into the vessel at a rate of $1.5 \mathrm{ml}$ per minute. Initiator was split into six equal portions and then added at half hour intervals during the monomer feeds. Throughout the process the reaction temperature was maintained at $80{ }^{\circ} \mathrm{C}$, however, once the feeds were completed, the temperature was raised to 83 ${ }^{\circ} \mathrm{C}$ and allowed to stabilise for 15 minutes. A final portion of initiator was then added and the reaction temperature was maintained at $83{ }^{\circ} \mathrm{C}$ for a further 75 minutes, after which the latex was cooled. The latex was filtered through a mesh in order to remove large aggregates.

Particle size was controlled through the surfactant concentration. Portions $(100 \mathrm{~g})$ of filtered latex were decanted and the $\mathrm{pH}$ of the latex was adjusted to 8.5 using $25 \mathrm{wt} . \%$ ammonium hydroxide solution. Aliquots (10ml) of the ADH (10 wt.\%, 5 wt.\% or 2 wt.\% aqueous solution) crosslinking agent were thoroughly stirred into portions of the latex. These concentrations correspond to the molar ratios summarised in Table 1.

Table 1: Molar ratios of ADH crosslinker to DAAM groups in Standard latex and the number of DAAM groups that can be crosslinked as a function of the wt.\% crosslinker in the latex.

\begin{tabular}{|c|c|c|}
\hline $\begin{array}{c}\text { ADH wt/\% } \\
\text { added to latex }\end{array}$ & $\begin{array}{c}\text { Number of moles ADH:1 mole } \\
\text { DAAM }\end{array}$ & $\begin{array}{c}\text { Number of crosslinkable } \\
\text { DAAM's per copolymer } \\
\text { molecule }\end{array}$ \\
\hline 1 & 0.37 & 6 \\
\hline 0.5 & 0.18 & 3 \\
\hline 0.2 & 0.075 & 1 \\
\hline
\end{tabular}

Mono-phasic latices with solids content of $40 \%$ were prepared with differing $\mathrm{T}_{\mathrm{g}}$ 's and particle sizes. The "standard" composition of latex had a $\mathrm{T}_{\mathrm{g}}$ of $\sim 52.5{ }^{\circ} \mathrm{C}$ and was made with particle sizes of 80,150 and $300 \mathrm{~nm}$. A latex with a $\mathrm{T}_{\mathrm{g}}$ of $118{ }^{\circ} \mathrm{C}$ was made using St (46.5 wt.\%), MMA (46.5 wt.\%), DAAM (5\% wt.\%), and MA (2 wt.\%) and will hereafter be referred to as the "high $\mathrm{T}_{\mathrm{g}}$ latex". A latex with $\mathrm{T}_{\mathrm{g}}$ of $-2.4{ }^{\circ} \mathrm{C}$ was made using BA (69.2 wt.\%), MMA (21.7 wt.\%), DAAM (7.2 wt.\%), and MA (1.9 wt.\%) and will hereafter be referred to as the "low $\mathrm{T}_{\mathrm{g}}$ latex". 


\section{Fundamentals of Keto-Hydrazide Crosslinking}

The fundamentals of the keto-hydrazide reaction were studied using model compounds as previously described. Solutions of 2-heptanone $(0.1 \mathrm{M})$ and octanoic hydrazide $(0.1 \mathrm{M})$ were prepared in methanol and aliquots combined. The reaction solutions were analysed using Fourier Transform Infra Red spectroscopy (Mattson Research Series), Nuclear Magnetic Resonance Spectroscopy (Jeol EX90) and Gas Chromatography Mass Spectroscopy (Thermo Finnigan Trace) in order to establish the nature of the reaction product and the catalytic influence of $\mathrm{pH}$.

\section{Chemical Crosslinking During Film Formation}

The change in surface $\mathrm{pH}$ of drying films was measured using $\mathrm{pH}$ indicator paper at the surface as a function of time until the touch-dry point. Gravimetric gel fraction measurements were performed on $1 \mu \mathrm{m}$ films bar-cast on silicon substrates via the extraction of the soluble portion using acetone. The change in percentage gel with drying time was measured as a function of particle size, film thickness and level of cross-linker.

An Atomic Force Microscope (AFM) (Veeco Dimension 3100) using ultra sharp silicon tips, of resonant frequency $130-250 \mathrm{kHz}$, and spring constant $48 \mathrm{~N} /$, was used in intermittent contact (tapping mode) to probe the extent and rate of particle flattening of latex films cast on to glass microscope slides. Unless stated otherwise, film formation was in still air at a temperature of $22{ }^{\circ} \mathrm{C}$.

\section{Characterisation of Surface Residues Evolved During Latex Film Formation}

The solubility of the surface residue on latex films was established by probing its solubility by comparing AFM scans before and after rinsing with water. X-ray Photoelectron Spectroscopy (XPS) provided surface sensitive chemical information. Cast films were analysed in order to determine the chemical composition of the residue and verify the nature of the film surface.

\section{Factors Influencing Surfactant Exudation During Film Formation}

During film formation, the humidity was adjusted through the use of saturated salt solutions in a closed container, and through air flow above the film. Film formation at a temperature of $9{ }^{\circ} \mathrm{C}$ was achieved in a refrigerator. Surfactant exudation was studied using AFM height and phase images, and probed as a function of latex $T_{g}$, temperature, crosslinking (with and without $\mathrm{ADH}$ ), humidity and evaporation rate.

\section{RESULTS \& DISCUSSION}

\section{Fundamentals of Keto-Hydrazide Crosslinking}

FTIR analysis revealed that 2-heptanone exhibits a strong carbonyl peak at $1710 \mathrm{~cm}^{-1}$ and octanoic hydrazide exhibits a strong band at $1628 \mathrm{~cm}^{-1}$ attributable to the amide carbonyl and $\mathrm{N}-\mathrm{H}$ stretching. On reaction, it would be expected that the intensity of the ketone carbonyl and the N-H stretching and bending peaks would be reduced, as they are consumed during crosslinking.

On reaction of the model components, a new peak was observed in the region $1670 \mathrm{~cm}^{-1}$. Figure 2 shows that with an excess of 2 -heptanone (2:1 molar ratio) a new 
peak is observed adjacent to the ketone carbonyl. As the level of octanoic hydrazide is low, indicated by the low intensities of the relevant peaks, then this new peak is unlikely to be related to the hydrazide. For a reaction mixture containing equimolar proportions of reactants, the ketone carbonyl is partially masked by the new peak. In the presence of excess octanoic hydrazide (2:1 molar ratio) the new peak appears adjacent to the amide peak. This would seem to rule out any possibility that the new peak is the result of an amide solution shift. Critically, the new peak at $\sim 1670 \mathrm{~cm}^{-1}$ is in the correct region for a reaction product containing $C=N\left(1690-1630 \mathrm{~cm}^{-1}\right)^{41}$.

Figure 2: An overlay of Infra Red showing the presence of an absorption band at $1670 \mathrm{~cm}^{-1}$ in the anticipated region for $\mathrm{C}=\mathrm{N}$ Imine. Spectrum 1 (red line): 2heptanone; Spectrum 2 (green line): excess 2-heptanone plus octanoic hydrazide; Spectrum 3 (purple line): Octanoic Hydrazide; Spectrum 4 (blue line): excess octanoic hydrazide plus 2-heptanone.

The ${ }^{13} \mathrm{C}$ NMR spectrum of 2-heptanone exhibits a peak at around $210 \mathrm{ppm}$, due to the ketone $\mathrm{C}=\mathrm{O}$ group. In comparison, the amide ester carbonyl in octanoic hydrazide is observed at around $175 \mathrm{ppm}$. On reaction the peaks due to the model component are depleted in acidic and neutral solutions as would be expected. Two new peaks are observed at around $160 \mathrm{ppm}$ and $150 \mathrm{ppm}$. The chemical shift for $\mathrm{C}=\mathrm{N}$ is expected in the 165 to $145 \mathrm{ppm}$ range ${ }^{41}$, and so this indicates that the product is an imine. There are no peaks present in the $140-100 \mathrm{ppm}$ region, where $\mathrm{C}=\mathrm{C}$ would be expected to be observed. There is evidence in the literature to indicate that the two peaks observed in the NMR spectrum are most probably due to

- the result of hydrogen bonding, due to methyl group influence, and 
- rotation about the $\mathrm{C}=\mathrm{N}$ bond, altering the field effect on the amide carbonyl, and thereby giving rise to two isomeric forms, as illustrated in the inset in figure 3 .

Figure 3: ${ }^{13}$ Carbon Nuclear Magnetic Resonance spectrum for reaction product between 2-heptanone and octanoic hydrazide under acidic conditions. The spectrum shows the depleted $\mathrm{C}=\mathrm{O}$ peak at $210 \mathrm{ppm}$ and four peaks in the $150-180 \mathrm{ppm}$ region attributed to the amide $\mathrm{C}=\mathrm{O}$ group and the $\mathrm{C}=\mathrm{N}$ group. Two peaks appear for each of these groups due to the possibility of two rotamer forms (structures shown inset).

Analysis of the product of the model reaction using GC/MS Chemical Ionisation highlighted the presence of a molecular ion of $255 \mathrm{amu}(\mathrm{M}+1)$, as would be anticipated from options (1) and (2) in Figure 1.

GC/MS analysis of alkaline solutions showed the presence of strong peaks for the reactants, 2-heptanone and octanoic hydrazide. In contrast, neutral and acidic solutions show a dominant product peak and diminished reactant responses. Comparison of the initial rates of the reactions under different $\mathrm{pH}$ condition clearly show that the chemical reaction rate increases with decreasing $\mathrm{pH}$, as illustrated in figure 4 . We conclude then that the reaction is acid catalysed. 
Figure 4: Graph showing the relationship between solution $\mathrm{pH}$ and initial reaction rate for octanoic hydrazide and 2-heptanone.

It is known that in a carbonyl group the electronegative oxygen withdraws electrons from the carbon bond resulting in the carbonyl carbon attaining a relative positive charge ${ }^{42}$. The electrophilic character of the carbon therefore significantly enhances its susceptibility to nucleophilic attack by electron-rich reagents ${ }^{42-47}$. Moreover, the part of the molecule immediately surrounding the carbon is flat, with oxygen, carbon and the two atoms directly bonded to the carbon lying in the same plane. Consequently, this part of the molecule is open and relatively unhindered from attack from above or below, in the direction perpendicular to the plane of the carbonyl group $^{45}$. As nitrogen exhibits electronegative character, and will readily donate a pair of electrons, it is expected that the carbonyl group will be prone to react with amines. The literature maintains that amines will react with both aldehydes and ketones, with the nature of the product being dependent on the species involved and reaction conditions ${ }^{46}$.

The susceptibility of the carbonyl group to nucleophilic attack is reduced by its attachment to electron-releasing alkyl groups that reduce the degree of positive charge on the carbon 42,43 . Therefore, as ketone structures comprise two alkyl groups attached to the carbon, compared to alkyl and hydrogen in an aldehyde molecule, then ketones would be expected to be less reactive than aldehydes. Indeed the presence of 
two alkyl groups would also be expected to incur some steric hindrance to attack on the carbon. It has been reported elsewhere that primary amines produce imines on reaction with aldehydes and ketones, although ketones react more slowly than aldehydes, sometimes requiring higher temperatures and longer reaction times 46,47 . In addition the progression of the reaction is inhibited by the formation of water, and the literature identifies that, in reaction studies, the water must be removed either by distillation or with a drying agent ${ }^{46}$. This latter behaviour is of particular interest in the case of the waterborne latex. It would seem reasonable to propose then that the presence of water will "block" the crosslinking reaction until, after sufficient drying has occurred. The water content of the film reaches a critical level where the equilibrium of the keto-hydrazide is shifted in favour of crosslinking.

Whilst primary amines and carbonyl groups will form imines, it is known that enamines can be formed on the reaction of aldehydes and ketones with secondary amines ${ }^{46}$. Furthermore, for carbonyl compounds with an $\alpha$-proton, a rotamer equilibrium can exist between the imine and the enamine, but in which the imine form predominates ${ }^{48}$. Despite the abundance of literature supporting imine formation from reaction between the carbonyl group and primary amines, specific reports on the ketohydrazide are few.

The literature indicates that the reaction of a nucleophile with a carbonyl compound is often catalysed by acid $43,45,46$. Protonation of the carbonyl oxygen further increases the positive charge at the carbon, making it much more susceptible to nucleophilic attack. So, in the case of a carbonyl compound nucleophilic addition will be favoured by high acidity. However, protonation of the amine produces a species lacking unshared electrons resulting in the loss of nucleophilic character. Thus, with respect to the amine compound, addition is favoured by low acidity. In reality, an efficient reaction condition would be a compromise, with the exact requirements depending on the reactivity of the carbonyl, and the basic character of the reagent.

Based on the experimental results of this research work and literature evidence, we propose the mechanism shown in figure 5 for the keto-hydrazide reaction in our latex system. 
Figure 5: Proposed mechanism for the keto-hydrazide reaction within the waterborne coating yielding an imine - not an enamine as has been proposed elsewhere.

In an acid environment the process commences with the formation of a bond between $\mathrm{H}^{+}$and the carbonyl oxygen. The nucleophilic amine group on the hydrazide molecule attacks the electrophilic carbonyl carbon, resulting in the formation of a C-N bond. Proton transfer occurs from the positively charged nitrogen to form a bond between $\mathrm{H}^{+}$and the oxygen, yielding a carbinolamine. Cleavage of the positively charged $\mathrm{H}_{2} \mathrm{O}$ from the central carbon then occurs, forming a resonance stabilised intermediate. The nitrogen stabilised carbocation is the conjugate acid of the imine and transfer of the hydrogen atom, attached to the nitrogen, to water yields the imine.

\section{Chemical Crosslinking During Film Formation}

Gravimetric measurement of latex solids fraction as a function of drying time during film formation was used as a rough indicator of the point of particle close packing. Parallel measurements of the wet film's $\mathrm{pH}$ with indicator paper revealed an increase in acidity over time, reaching a $\mathrm{pH}$ of $\sim 6$ after six minutes of drying. In a complementary experiment, litmus was added to the wet latex dispersion prior to film formation. This experiment clearly revealed that the film became significantly acidic when dried. The decreasing $\mathrm{pH}$ during film formation is attributed to the loss of ammonia through evaporation. Significantly, latex pre-neutralised with non-volatile amine did not exhibit the $\mathrm{pH}$ change.

Figure 6: Graph showing the change in $\mathrm{pH}$ for a thin latex film during film formation with respect to change in wt.\% solids. (As the densities of the water and copolymer are nearly identical wt.\% will be very close to vol.\%.)

Combining the $\mathrm{pH}$ measurement with the solids fraction measurements (Figure 6), we are then able to establish the solids fraction when crosslinking is favoured. The figure shows that the $\mathrm{pH}$ decreases below 7 when a solids content of approximately $68 \mathrm{wt} . \%$ is achieved. Depending on the particular packing of the particles, this solids content would correspond to the point of contact between particles. (For mono-size hard particles the maximum solids fraction at close packing is 74 vol.\%.) The gas chromatography data clearly showed that the chemical reaction rate increases 
substantially below a $\mathrm{pH}$ of 7 . Therefore crosslinking in the film is favorable only near and after the point of close packing. Polymer interdiffusion likewise cannot occur until particles have made physical contact, of course. Thus, interdiffusion and crosslinking will probably occur simultaneously. As already noted there is an optimum $\mathrm{pH}$ to achieve the fastest reaction rate, and it is expected that the acidity in the polymer phase will be correlated with that in the water phase.

The influence of the level of ADH cross-linker on the polymer gel fraction is shown in figure 7. Gel content increases rapidly over the first 15 minutes of film drying, after which the rate slows but never reaches $100 \%$. The rate of increase in gel content and the final gel value are identical for $0.5 \mathrm{wt} . \%$ and $1 \mathrm{wt} . \% \mathrm{ADH}$, indicating that 0.5 wt.\% is sufficient to ensure that $\mathrm{ADH}$ reacts with at least one DAAM group per molecule. Hence a film containing $0.2 \mathrm{wt} . \%$ achieves a lower gel fraction, being insufficient for the crosslinking of every polymer molecule. In order to investigate this observation further, films subjected to vacuum drying rather than drying in air, were measured also. An increase of around 2 to $3 \%$ (not shown) in the final gel fraction was found, but $100 \%$ gel was still not attained. These results indicate the presence of some residual water presence in air-dried films.

Possible - but unlikely - explanations for why the maximum gel fraction is only around $90 \%$ are that some macromolecules do not contain DAAM or that some molecules only have one crosslink. Another possibility is that there is some intramolecular crosslinking in which two DAAM groups on the same molecule are reacted with an ADH cross-link molecule. Recent simulations of gel fraction [26], which consider the effects of molecular weight polydispersity and the number of crosslink units, offer the most likely explanation.

Statistical variation means that some polymer molecules will have greater than average crosslinks per molecule, whilst others will have less than the average or even zero. Simulations [26] show that as polydispersity increases then more crosslinked units are required to achieve a given gel fraction. Thus, a lower polydispersity favours a higher gel fraction for a given number of crosslinks. The "standard" latex has a polydispersity of $\sim 2.7$. When $1 \mathrm{wt} . \% \mathrm{ADH}$ is added it is estimated that there can be up to 6 crosslinks per polymer molecule. In this case it is estimated that approximately $90 \%$ gel can be achieved, as was observed in the experiments.

To achieve one crosslink per polymer molecule, one half of a molecule of crosslinker is required for each polymer molecule. On average each "standard" latex polymer molecule contains sixteen DAAM groups. Using the previously calculated molar ratio values, Table 1 , the number of DAAM groups that can actually be reacted may be calculated. For 1 wt.\% ADH added to "standard" latex, there are 0.37 moles of ADH per 1 mole of DAAM. It follows then that $37 \%$ of the available DAAM groups can be reacted, corresponding to 6 crosslinks per polymer molecule. In the case of 0.5 wt.\% and 0.2 wt.\% ADH "standard" latex this reduces to 3 and 1 crosslinks per polymer molecule, respectively. Clearly this indicates that there is insufficient ADH crosslinker present to react with all of the available DAAM groups. The maximum crosslink density cannot be achieved in these formulations, and furthermore ADH is not soluble in water at higher concentrations. 
Gel fraction measurements (Figure 7) also indicate that the latex particle size influences the rate of increase of the gel content. The gel fraction of latex comprising $80 \mathrm{~nm}$ particles reaches a plateau value after around 15 minutes. However, the rate of gelation becomes slower with increasing latex particle size $(150 \mathrm{~nm}$ and $300 \mathrm{~nm})$. In order to achieve the maximum gel fraction, the ADH must diffuse a distance on the order of the particle radius. The dependence of gelation rate on the particle size indicates that the reaction is limited by the diffusion of $\mathrm{ADH}$ into the latex particles. Our results indicate that the $\mathrm{ADH}$ is partitioned mainly in the aqueous phase initially and enters the polymer phase during the later stages of film formation

Figure 7: Gel Fraction graphs showing the influence of level of cross-linker (top), film thickness (top) and particle size (lower) on the rate of gel evolution in the latex film.

The film thickness of the latex dispersion was varied in order to probe the influence of water content on the progression of the crosslinking reaction. It has been discussed previously in this work that the keto-hydrazide reaction is inhibited by the presence of water. Therefore that keto-hydrazide crosslinking in a latex film is not expected to occur unless a sufficient fraction of the water component has evaporated. By studying films with different thickness the quantity of water per unit area required to evaporate was therefore varied. It was observed that the onset of crosslinking is delayed in 
thicker films, presumably due to the presence of more water per unit area. This effect is particularly evident in the first 20 minutes of drying. Furthermore, the rate of crosslinking in thicker films appears to be slower, which could be attributed to "skin formation" in the thicker film slowing down water evaporation and $\mathrm{pH}$ changes in the later stages of drying.

The AFM topographic images in Figure 8 show that in films from the high $\mathrm{T}_{\mathrm{g}}$ latex with $\mathrm{ADH}$ cross-linker (8A and $8 \mathrm{~B}$ ), particles retain their particle identity. The surface does not exhibit significant particle flattening, and particle boundaries remain. In the absence of $\mathrm{ADH}$, the film structure is the same (images not shown). Hence, in this case, it is the particle rigidity itself, and not the crosslinking, that is resisting particle flattening and interdiffusion. In contrast, the low $\mathrm{T}_{\mathrm{g}}$ latex with $\mathrm{ADH}$ crosslinker (8C and 8D) rapidly forms a significantly flatter film. Interestingly, a honeycomb structure was retained at the surface with boundaries apparent, as shown in Figure $8 \mathrm{D}$. This structure is not observed in cross-linker-free low $\mathrm{T}_{\mathrm{g}}$ latex films $(8 \mathrm{E}$ and $8 \mathrm{~F}$ ) which exhibited essentially instantaneous flattening of the surface particles and in which no particle boundaries are apparent. These results show that crosslinking inhibits particle flattening and retards interdiffusion.

Film formation of the low $\mathrm{T}_{\mathrm{g}}$ latex in the absence of cross-linker is accompanied by the development of a surface residue of $\sim 5 \mathrm{~nm}$ in thickness, which spreads laterally as drying time increases. Figure 9 (images 9A-D) shows the growth of the residues with time. At later times this residue appeared to aggregate and reticulate into isolated "blobs". This observation prompted further investigation.

Films were subjected to XPS analysis that established the chemical composition of the residue to be consistent with surfactant. After rinsing the film surface with water, sodium and sulphur, consistent with sodium lauryl sulphate, were not detected with XPS, which is expected for water-soluble surfactant. Figure $9 \mathrm{E}$ and $9 \mathrm{~F}$ shows the corresponding surface structure after rinsing, in which no residue is observed. When cross-linker is added to the low $\mathrm{T}_{\mathrm{g}}$ latex, XPS and AFM analysis find no evidence for surfactant excess at the film surface. Hence crosslinking inhibits the exudation of surfactant. After rinsing, surfactant continued to exude to the surface of the low $\mathrm{T}_{\mathrm{g}}$ latex, as revealed in Figure $9 \mathrm{G}$ and $9 \mathrm{H}$. As surfactant was not detected at the surface of the high $\mathrm{T}_{\mathrm{g}}$ latex, particle deformation is likely to be a key factor, prompting further investigation discussed below. 
Published in Journal of Coatings Technology Research (2008) 5, 285-297.

Figure 8: AFM topographic images showing the dependence of film structure on polymer $\mathrm{T}_{\mathrm{g}}$, crosslinking and film formation time. The left column show the structure after one hour and the right column shows the structure after 24 hours. Images A and $\mathrm{B}$ : high $\mathrm{T}_{\mathrm{g}}$ latex plus $\mathrm{ADH}$ cross-linker; $\mathrm{C}$ and $\mathrm{D}$ : low $\mathrm{T}_{\mathrm{g}}$ latex plus $\mathrm{ADH}$ cross-linker; $\mathrm{E}$ and F: as-prepared low $\mathrm{T}_{\mathrm{g}}$ latex. All images are $1.5 \mu \mathrm{m} \times 1.5 \mu \mathrm{m}$

\section{Factors Influencing Surfactant Exudation During Film Formation}

In order to explore the influence of particle deformation on the rate of surfactant exudation, low $\mathrm{T}_{\mathrm{g}}$ latex films were subjected to drying at $\mathrm{T}<\mathrm{T}_{\mathrm{g}}$ of the polymer prior to AFM imaging at room temperature. The topography and phase AFM images in Figures 10A-C show that surfactant exudation is inhibited in comparison to films dried at room temperature $\left(T>T_{g}\right)$, shown already in Figures 9A-D. When the cooled films were raised to room temperature, the evolution of surfactant domains were then observed, albeit at a lower surface coverage compared to the film that had been 
formed at room temperature. Figure 11 shows some quantitative analysis of the surfactant exudation phenomenon in which the surface area of the surfactant regions is estimated from AFM images. The surface area of the surfactant increases approximately at a constant rate in all cases, but the rate is heavily dependent on the particle deformability. Harder particles, as obtained here when $\mathrm{T}<\mathrm{T}_{\mathrm{g}}$, have a lower rate of surfactant exudation in comparison to softer particles that readily coalesce. Holl and co-workers ${ }^{49}$ reached a similar conclusion when they compared surfactant exudation from two latices having different $T_{g}$ values. In their experiment, the differences in surfactant exudation might also have been explained by differences in chemical composition. In our experiments, on the other hand, the same latex is being used, so the difference in exudation rate cannot be attributed to chemical differences. Our results could be explained by a co-operative mechanism between particle deformation and surfactant exudation. When there is flattening at particle/particle boundaries, surfactant might be pushed away from that surface. 
Published in Journal of Coatings Technology Research (2008) 5, 285-297.

Figure 9: AFM micrographs (topographic images on the left and phase images on the right) of low $\mathrm{T}_{\mathrm{g}}$ latex without cross-linker showing the evolution of surface residue (attributed to sodium lauryl sulphate surfactant). A and B: latex film one hour after film casting; $C$ and D: latex film 6 hours after casting; $E$ and F: the same latex as $C$ and $\mathrm{D}$ after rinsing; $\mathrm{G}$ and $\mathrm{H}$ : the rinsed latex after aging for a further 24 hours. All AFM images are $5 \mu \mathrm{m} \times 5 \mu \mathrm{m}$. 
Published in Journal of Coatings Technology Research (2008) 5, 285-297.

Figure 10: AFM phase images showing the evolution of SLS surfactant to the surface of a low $\mathrm{T}_{\mathrm{g}}$ latex with time as a function of temperature and humidity. The age of the films in the first column is 90 minutes; second column 6 hours; third column 3 days. A-C: film formed at $9{ }^{\circ} \mathrm{C}$ and $51 \%$ relative humidity; D-F: film formed at $22{ }^{\circ} \mathrm{C}$ and $13 \%$ relative humidity; G-I: film formed at $22{ }^{\circ} \mathrm{C}$ and $39 \%$ relative humidity; J-L: film formed at $22^{\circ} \mathrm{C}$ and $80 \%$ relative humidity. All AFM images are $5 \mu \mathrm{m} \times 5 \mu \mathrm{m}$. 
Published in Journal of Coatings Technology Research (2008) 5, 285-297.

Figure 11: The time-dependence of the surface coverage (in $\mu \mathrm{m}^{2}$ ) of the surfactant measured from analysis of AFM images. Films were stored at two different relative humidities (13\% and 39\%) at $22{ }^{\circ} \mathrm{C}$ (at top) and at two different temperatures $\left(9{ }^{\circ} \mathrm{C}\right.$ and $22{ }^{\circ} \mathrm{C}$ ) but similar humidity's (at bottom). After $5760 \mathrm{~min}$, the humidity was increased from $13 \%$ to $39 \%$ (top graph).

Further experiments were conducted in which the water evaporation rate during film formation was adjusted through the relative humidity. Recent modelling ${ }^{50}$ of surfactant distribution has predicted that slower evaporation rates will lead to a more uniform distribution of surfactant in the vertical direction. The AFM images in figures 10D-L show visually that low $\mathrm{Tg}$ latex films exhibit rates of surfactant exudation that are dependent on the evaporation rate. In a dry atmosphere $(9 \%$ 
relative humidity), wherein the water evaporation rate is fastest, surfactant exudation occurs very slowly and appears as small beads on the film surface. In comparison, a film dried at an intermediate humidity (39\%) shows a higher rate of exudation and the small domains rapidly aggregate to form larger "blobs". Finally, at high humidity $(80 \%)$ and slowest evaporation, the surfactant rapidly exudes to the surface forming extensive coverage. Figure 11 shows quantitatively how the humidity affects the surfactant exudation. This observed trend in surfactant coverage is the exact opposite of what is expected from the model ${ }^{50}$ that considers the surfactant transport in the aqueous phase. However, in these experiments not only is the evaporation rate varied (as considered in the model), but also the hydrophilicity at the film/air interface is increased with increasing relative humidity. The surfactant could be favoured at a more hydrophilic interface. Furthermore, water condensation in the surfactant layer could impart mobility to the surfactant.

Figure 12: AFM Phase images showing the influence of evaporation conditions on the exudation of SLS surfactant to a low $\mathrm{T}_{\mathrm{g}}$ latex film surface (17 hours old). A: After

film formation with fast evaporation under an air stream; B: film formation under normal evaporation conditions (39\% RH); C: after film formation with slower evaporation obtained with a partly covered enclosure; D: after film formation with the slowest evaporation, obtained within a closed container. All AFM images are $5 \mu \mathrm{m} \mathrm{x}$ $5 \mu \mathrm{m}$.

In order to explore this phenomenon further, low $\mathrm{T}_{\mathrm{g}}$ latex films were prepared under different drying conditions. Films subjected to an air stream, keeping the humidity low immediately above the film, were found to exhibit little surfactant exudation (Figure 12A). On removal of the air stream, surfactant domains evolved as for a film dried under normal conditions. Films dried in partly and completely enclosed conditions (Figures 12C and D), effectively creating a higher humidity immediately above the film, were found to exhibit rapid and extensive surfactant exudation and coverage that increased in area as the evaporation rate decreased.

\section{CONCLUSIONS}

Fundamentals of the keto-hydrazide reaction have been studied utilising a model system. The complementary use of Fourier transform infra red spectroscopy, NMR Spectroscopy, and Gas Chromatography/Mass Spectroscopy has provided supporting evidence to clarify - for the first time - that the reaction yields an imine. No data support the presence of an enamine in the product. NMR provides additional verification suggesting rotamer behaviour and hydrogen bonding of the product in solution. Moreover, the crosslinking reaction is acid catalysed and the reaction rate increases as $\mathrm{pH}$ decreases. Hence, in our ammonia neutralised latex the crosslinking 
reaction is favoured by the loss of water during drying and the simultaneous decrease in $\mathrm{pH}$ arising from the evaporation of ammonia. Crosslinking is certainly possible once the latex particles are close packed, and there is a rapid increase in gel content in a short period.

The crosslinking influences the later stages of film formation. The flattening of latex particles and interdiffusion leading to the blurring of particle/particle interfaces, as observed with AFM analysis, were both inhibited by crosslinking. The amount of particle deformability was identified as a key factor in the amount of surfactant exudation. Factors that increase the amount of particle deformation and coalescence (e.g. higher film formation temperature, lower latex $\mathrm{T}_{\mathrm{g}}$, and no crosslinking) promote surfactant exudation. Conversely, surfactant exudation is inhibited when particles are less deformable and slower to coalesce. In addition, a more hydrophilic atmosphere (high humidity) encourages surfactant segregation at the interface with the film. Exudation to a "clean surface" can be triggered by raising the temperature (thus increasing particle deformation) or by raising the humidity (thus raising the hydrophilicity at the interface).

\section{ACKNOWLEDGEMENTS}

We acknowledge Dr Ann Woollins for NMR support, Mr John Humphrey for support with GC/MS, Mr Steven O'Flynn and Mr Daniel Azzian for their practical help, Miss Junko Sano (Dainippon Ink and Chemical) for the XPS analysis, and Professor Stephen Davidson (University of Kent) for his useful comments on NMR and Imine chemistry.

\section{REFERENCES}

1. Kerhsaw, Y., Europ Coat J, 4/98, (1998), 230-232, 234, 235.

2. Steward, PA., Hearn, J., Wilkinson, MC., Adv in Colloid \& Interf Sci, 86, 195 (2000).

3. Provder, T., Urban, MW., Film Formation in Coatings, Mechanisms, Properties and Morphology, ACS Symposium Series 790, Oxford University Press, vii, (2001).

4. Keddie, JL., Film formation of latex, Materials Science \& Engng Reports, R21 (3), (1997).

5. El-Aasser, M., Sudol, ED., Emulsion Polymerisation and Emulsion Polymers, Eds PA Lovell, MS El-Aasser, John Wiley \& Sons, Chapter 2, (1997).

6. Hellgren, AC., Wallin, M., Weissenborn, PK., McDonald, PJ., Glover, PM., Keddie, JL., Progress in Organic Coatings, 43, 85, (2001).

7. Aradian, A., Raphael, E., De Gennes, PG., Macromolecules, 33, 9444, (2000).

8. Athey, RD., European Coatings Journal, 816, (1996).

9. Buckmann, F., Overbeek, A., Nabuurs, T., European Coatings Journal, 6, 53, (2001).

10. Pinenq, P., Winnik, MA., J Coat Technol, 72 (903), 45 (2000).

11. Winnik, MA., J Coat Technol, 74 (925), 49, (2002).

12. Hanrahan, B., Hakko, K., Murayama, T., Hotta, I., FSCT Midyear Symposium: Crosslinking for coatings - Meeting the challenges. Self-crosslinking emulsions for improved chemical resistance based on the reaction of diacetone acrylamide with dihydrazides, Orlando, (2004). 
13. Bakker, P., Mestach, D., Surface Coatings International Part B: Coatings Transactions, 84 (B4), 243, (2001).

14. Lee, SB., Billiani, J., Pfohl, WF., Lee, KI., Proceedings of $30^{\text {th }}$ International Waterborne High Solids and Powder Coatings Symposium, Waterborne 1K Self cross-linkable acrylic core-shell polymers, New Orleans, (2003).

15. Illsley, DR., Sun Chemical Ltd, Products \& New Materials Confidential Laboratory Report: Current and emerging themes in polymer science, (2003).

16. Esser, RJ., Devona, JE., Setzke, DE., Wagemans, L., Progress in Organic Coatings, 36, 45, (1999).

17. Zosel, A., Ley, G., Macromol, 26, 2222, (1993).

18. Pinenq, P., Winnik, MA., J Coat Technol, 72, 45, (2000).

19. Zosel, A., Ley, G., Polymer Bulletin, 27, 459, (1992).

20. Pinenq, P., Winnik, MA., Ernst, B., Juhue, D., J Coat Technol, 72 (903), 45, (2000).

21. Tamai, T., Pinenq, P., Winnik, MA., Macromol, 32, 6102, (1999).

22. Daniels, ES., Klein, A., Progress in Organic Coatings, 19, 359, (1991).

23. Brown, WT., J Coat Technol, 72 (904), 63, (2000).

24. Winnik, MA., Pinenq, P., Kruger, C., Zhang, J., Yaneff, PV., J Coat Technol, 71 (892), 47, (1999).

25. Winnik, MA., J Coat Technol, 74 (925), 49, (2002).

26. Taylor, JW., Winnik, MA., JCT Research, 1 (3), 163, (2004).

27. Richard, J., Wong, K., J Polymer Sci: Part B: Polymer Physics, 33, 1395, (1995).

28. Yoo, JN., Sperling, LH., Glinka, CJ., Klein, A., Macromolecules, 23, 3962, (1990).

29. Sperling, LH., Klein, A., Sabisivam, M., Kim, KD., Polymers for Advanced Technologies, 5, 453, (1994).

30. Bradford, EB., Vanderhoff, VW., J Macomol Chem, 1 (2), 335, (1966).

31. Bradford, EB., Vanderhoff, VW., J Macomol Sci Phys, B6 (4), 671, (1972).

32. Juhue, D., Wang, Y., Lang, J., Leung, OM., Goh, CM., Winnik, MA., J Polymer Science: Part B: Polymer Science, 33, 1123, (1995).

33. Du Chesne, A., Gerharz, B., Lieser, G., Polymer International, 43, 187, (1997).

34. Tzitzinou, A., Jenneson, PM., Clough, AS., Keddie, JL., Lu, JR., Zhdan, P., Treacher, KE., Satguru, R., Progress in Organic Coatings, 35, 89, (1999).

35. Belaroui, F., Hirn, MP., Grohens, Y., Marie, P., Holl, Y., J of Colloid and Interface Sci, 261, 336, (2003).

36. Hellgren, AC., Weissenborn, P., Holmberg, K., Progress in Organic Coatings, 35 , 79, (1999).

37. Butler, LN., Fellows, CM., Gilbert, RG., J of Applied Polymer Science, 92, 1813, (2004).

38. Butler, LN., Fellows, CM., Gilbert, RG., Progress in Organic Coatings, 53, 112, (2005).

39. Aramendia, E., Barandiaran, MJ., Grade, J., Blease, T., Asua, JM Langmuir, 21, 1428, (2005).

40. Charmeu, JY., Kientz, E., Holl, Y., Progress in Organic Coatings, 27, 87, (1996).

41. Williams, DH., Fleming, I., Spectroscopic Methods in Organic Chemistry, $3^{\text {rd }}$ Edition, McGraw-Hill Book Company (UK) Limited, (1980).

42. Murray, PRS., Advanced Chemistry, Pan Books Ltd, Chapter 36, (1987).

43. Howarth, J., Core Organic Chemistry, John Wiley \& Sons Ltd, Chapter 10, (1998).

44. Patai, S., The Chemistry of the Carbonyl Group, Interscience Publishers, Chapter 1, (1966). 
Published in Journal of Coatings Technology Research (2008) 5, 285-297.

45. Morrison, FT., Boyd, RN., Organic Chemistry, $4^{\text {th }}$ Edition, Allyn \& Bacon Inc. Chapter 18, (1983).

46. Patai, S., The Chemistry of the Carbon-Nitrogen Double Bond, Interscience Publishers, Chapter 2, (1970).

47. Graham Solomons, TW., Organic Chemistry, $3^{\text {rd }}$ Edition, John Wiley \& Sons Ltd, (1993).

48. Furniss, BS., Hannaford, AJ., Smith, PWG., Tatchell, AR., Vogel's Textbook of Practical Organic Chemistry, $5^{\text {th }}$ Edition, Longman Scientific \& Technical, Chapter 5, (1989).

49. Belaroui, F., Cabane, B., Dorget, A., Grohens, Y., Marie, P., Holl, Y., J. Colloid \& Interf. Sci. 262, 409, (2003).

50. Gundabala, VR., Zimmerman, WB., Routh, AF., Langmuir, 20, 8721, (2004). 